\title{
Discord of Biological and Psychological Measures in a Group of Depressed African American and White Cancer Patients
}

\author{
Amy Y. Zhang* \\ Frances Payne Bolton School of Nursing, Case Western Reserve University, 10900 Euclid Avenue, Cleveland, OH \\ 44106-4904, USA
}

\begin{abstract}
Objective: This study examined racial differences in the self-report of depressive symptoms by reference to biological states.

Methods: The study used a convenience sample of 20 depressed cancer patients (CES-D $\geq 16$ ) (15 African Americans and 5 Whites). Subjects completed depression assessment on a battery of psychological measures and provided blood and saliva samples. Laboratory tests were performed on biomarkers (serotonin, cortisol and IL-6). T-test was computed to examine racial differences on biological and psychological measures.

Results: Depressed Whites had a significantly higher cortisol level than depressed African Americans, but no significant group difference was found on any self-reported psychological measures of depression. There was a trend that African Americans reported fewer depressive symptoms on psychological measures but exceeded Whites on the domain of somatization; however, such group differences did not approach statistic significance in this small sample.

Conclusion: African Americans did not appear to underreport depression in consideration of their biological states, but had a tendency to report more somatic symptoms than Whites; this may be attributable to non-depression diseases or reporting behavior rather than somatic sensitivity. African Americans exhibited more mistrust in the health care system, which could affect the self-report of depression. There is a discord between biological and psychological measures of depression. Biomarkers prove to be useful for evaluating racial difference in the self-report of depression.

Implication for Nursing: Nurses should be cautious of somatic complaints when assessing African American cancer patient's depression. Establishing trust is essential for an accurate assessment of depression in African American cancer patients.
\end{abstract}

Keywords: African American, biomarker, cancer, cortisol, depression, race.

\section{INTRODUCTION}

Depression is a significant predictor of cancer survival $[1,2]$. African Americans have the highest cancer mortality and shortest survival after receiving a cancer diagnosis among all racial and ethnic groups in the United States [3]. Understanding racial difference in self-report is essential for improving depression assessment in this underserved population.

Existing literature suggests a racial difference in the presentation of depressive symptoms. Depressed African Americans complained more frequently than depressed white Americans about somatic symptoms, including poor appetite, fatigue, psychomotor retardation and sleep disturbance [4, 5]. The findings may indicate that African Americans have heightened somatic sensitivity or verbally amplify physical sickness sensations in self-report [6]. A competing theory, however, is that depressed African Americans are sicker than depressed white Americans due to an unequal burden of chronic illness [7].

\footnotetext{
*Address correspondence to this author at the Frances Payne Bolton School of Nursing, Case Western Reserve University, 10900 Euclid Avenue, Cleveland, OH 44106-4904, USA; Tel: (216) 368-0968;

Fax: (216) 368-3542; E-mail: axz16@case.edu
}

The literature has also shown that depressed African Americans report fewer negative emotions than depressed white Americans [4, 8]. For example, Gary and Yarandi reported [2004] that the factor loading of affective items in the BDI-II was lower than that of somatic and cognitive items among 206 healthy AA women. Brown et al. [9, 10] described depressed moods to be not as blatant as other depressive symptoms in AA women. Whether this observed "flat affect" in African Americans is an accurate account or a filtered presentation of biopsychological states remains unknown.

To assess racial difference in the manifestation of depressive symptoms by reference to the biological underpinnings is important, particularly for patients with a malignant tumor. Cancer-related symptoms including fatigue, decreased psychomotor activity, poor sleep and change in appetite resemble vegetative symptoms of depression $[11,12]$, making the detection of depression more difficult. Modern science has identified a number of biological markers for assessing depression. Low serotonin has been earmarked for depressive emotions [13]; high cortisol is frequently used as an indicator of mental stress [14]. On the other hand, high plasma Il-6 concentration and immunosuppression [e.g., decreased NK cell activity and 
proliferation responses of lymphocytes] were reported to be associated with depression in cancer patients $[15,16]$. These biomarkers can provide a biological baseline for evaluating racial differences in the self-report of depressive symptoms. Therefore, we collected biomarkers from a small group of cancer patients who were participating in a study of cancerrelated depression. The goal was to examine racial differences in biological and psychological measures to shed light on the self-report of depression.

\section{MATERIALS AND METHODOLOGY}

The study was conducted in Cleveland, Ohio in 2008 with institutional IRB approval. Subject eligibility included a diagnosis of early-stage (I-III) breast or prostate cancer within the past 3 years, having completed cancer treatments for at least 6 months and not having a psychotic disorder. Having identified potentially eligible patients through a hospital tumor registry and obtaining physicians' permission, an introductory letter and consent were mailed to the patients. Research staff followed this up with a call to introduce the study and used the Center for Epidemiologic Studies-Depression Scale (CES-D) to screen for depression. Seventy-five patients were recruited for a parental study of depression, of whom 30 depressed participants (CES-D $\geq 16$ ) were invited to provide biological specimen and 21 consented at a rate of $70 \%$. Fifteen African Americans and five Whites completed study procedure.

At a scheduled face-to-face interview, written consent was obtained and standard depression instruments were administered including the Hamilton Rating Scale for Depression (HAM-D), a commonly used and observer-rated 17-item scale; the Beck Depression Inventory-II (BDI-II), a 21-item self-rating scale known for its emphasis on affective symptoms of depression; the Brief Symptom Inventory (BSI), a 53-item self-rating symptom scale; and the Group-Based Medical Mistrust Scale (GBMMS), a 12-item scale that assesses medical mistrust in health care system and treatment provision.

Blood was drawn in the hospital immediately after the completion of questionnaires. The obtained blood sample was centrifuged before it was sent to a laboratory. The participants were taught how to obtain a saliva sample and provided with a testing kit. They took a saliva sample of free cortisol between 7-9 a.m. and then between 9-11 p.m. at home, stored them in a fridge and returned them the next day using a provided mailing label. Laboratory tests were performed on blood and saliva samples in the hospital to measure serotonin, cortisol and IL-6. The participants received $\$ 35$ for completing the interview and $\$ 30$ for providing biological specimen.

Two research assistants entered data independently using the Statistical Package for the Social Sciences (SPSS) version 19.0. Any discrepancies between the two SPSS data files were resolved by checking the original coding on the hard copy to ensure data entry consistency.

\section{RESULTS}

T-test and Fisher's exact test did not detect any significant difference between depressed African American and White groups in terms of age (mean age $=58$ or 59 , respectively), gender ( $40 \%$ vs $60 \%$ female), education $(73 \%$ vs $60 \%$ non-college), income $(46 \%$ vs $20 \%<\$ 50,000)$, cancer treatment ( $47 \%$ vs $100 \%$ for surgery, $67 \%$ vs $60 \%$ for radiation and $27 \%$ vs $20 \%$ for chemotherapy), a depression diagnosis prior to cancer $(20 \%$ vs 0$)$, comorbidity (mean number of disease $=7$ and 5 respectively), medications (mean number of medication $=6$ and 4 ) and pain in last 24 hours (mean rating $=5$ and 3 , respectively), despite some across-group variations. There was a tangible and insignificant difference between groups in the employment status $(60 \%$ vs $20 \%$ unemployed, $p=.11)$. The group difference in marital status was marginally significant $(p=$ $.07)$ with more Whites (80\%) than African Americans (27\%) being married. The lack of statistic significance was due to the small sample size and low statistic power (Table 1).

The level of morning cortisol was nearly twice as high in depressed Whites as in depressed African Americans, and this group difference was significant $(p=.036)$. There was a trend of lower serotonin, IL-6 and evening cortisol in the White group, but the group difference did not approach statistic significance.

The mean scores on psychological measures (CES-D, HAM-D, BDI-II, BSI and PSSI) were slightly higher in the White group, but not significantly different between groups. On the contrary, the African American group reported a significantly higher mean score on GBMMS-Suspicion domain $(p=.032)$ and slightly higher but not significantly different mean scores on the BSI-Somatic domain than the White group (Table 2).

\section{DISCUSSION}

The significantly higher level of morning cortisol suggests that the White group experienced severer acute stress than the African American group in this study. The psychological measures of depression, however, did not discern any significant group difference, demonstrating a discrepancy between biomarkers and psychological measures of depression. Perhaps psychological measures are less sensitive to biological states or not conducive to capturing acute stress. In any case, we did not find evidence of African Americans' underreporting of depressive symptoms, or the so-called "flat affect," because in contrast with biological findings, there was little racial difference in the self-report of depression on psychological measures.

Of note is the African American group's insignificant, slightly higher score on BSI's somatization subscale. In this study the African American group had lower morning cortisol and higher serotonin than the White group or, in other words, a better biological state; this contradicts their self-report of more somatic symptoms. It is inconceivable that African Americans' somatic complaint originated from heightened bodily sensations of depression since they experienced less stress, but the finding does not exclude the possibility that such a complaint was related to diseases other than depression.

Existing literature indicates greater mistrust of the health care system among African Americans [17]. Consistent with the literature, the African American group in this study reported significantly greater mistrust in the health care system and professionals than the White group. Medical mistrust has an origin in cultural belief and life experiences for African Americans [18]. It is plausible that the mistrust 
Table 1. Sample Demographics

\begin{tabular}{|c|c|c|c|c|c|}
\hline \multirow[t]{2}{*}{ Variable } & \multicolumn{2}{|c|}{$\begin{array}{l}\text { Depressed African } \\
\text { Americans }(n=15)\end{array}$} & \multicolumn{2}{|c|}{ Depressed Whites $(n=5)$} & \multirow[t]{2}{*}{$p$} \\
\hline & $\mathbf{N}$ & $\%$ & $\mathbf{N}$ & $\%$ & \\
\hline \multicolumn{6}{|l|}{ Gender } \\
\hline Female & 6 & $40 \%$ & 3 & $60 \%$ & .62 \\
\hline \multicolumn{6}{|l|}{ Marital status } \\
\hline Married & 4 & $27 \%$ & 4 & $80 \%$ & .07 \\
\hline Single & 3 & 20 & 1 & 20 & \\
\hline Other (widowed, divorced etc.) & 8 & 53 & 0 & 0 & \\
\hline \multicolumn{6}{|l|}{ Education } \\
\hline$\leq$ High school & 3 & $20 \%$ & 1 & $20 \%$ & .79 \\
\hline Some college & 8 & 53 & 2 & 40 & \\
\hline$\geq$ College & 3 & 20 & 2 & 40 & \\
\hline Unknown & 1 & 7 & 0 & 0 & \\
\hline \multicolumn{6}{|l|}{ Employment } \\
\hline Full time & 4 & $27 \%$ & 3 & $60 \%$ & .11 \\
\hline Half time & 0 & 0 & 1 & 20 & \\
\hline Unemployed & 9 & 60 & 1 & 20 & \\
\hline Unknown & 2 & 13 & 0 & 0 & \\
\hline \multicolumn{6}{|l|}{ Income (optional) } \\
\hline$<\$ 25000$ (household) & 5 & $33 \%$ & 0 & 0 & .20 \\
\hline$<\$ 50,000$ & 2 & 13 & 1 & $20 \%$ & \\
\hline$\geq \$ 50,000$ & 1 & 7 & 2 & 40 & \\
\hline Unknown & 7 & 47 & 2 & 40 & \\
\hline Prior depression diagnosis & 3 & $20 \%$ & 0 & 0 & .54 \\
\hline Had surgery & 7 & $47 \%$ & 5 & $100 \%$ & .14 \\
\hline Had radiation & 10 & $67 \%$ & 3 & $60 \%$ & 1.0 \\
\hline \multirow[t]{2}{*}{ Had chemotherapy } & 4 & $27 \%$ & 1 & $20 \%$ & 1.0 \\
\hline & Mean & S.D. & Mean & S.D. & $p$ \\
\hline Age & 58.2 & 7.2 & 59.2 & 9.7 & .81 \\
\hline Number of disease & 6.5 & 4.4 & 4.8 & 1.5 & .47 \\
\hline Number of medication & 5.6 & 2.5 & 3.6 & 3.6 & .18 \\
\hline Pain within 24 hours $(0-10 p t)$ & 5.1 & 3.1 & 2.8 & 2.8 & .17 \\
\hline
\end{tabular}

has an influence on African Americans' self-report of depression.

Overall, the observed discord between biological and psychological measures is not surprising but worth noting. Using biomarkers introduces a frame of reference for assessing racial difference in the self-report of depressive symptoms, particularly in cancer patients whose psychobiological states are impinged by both mental and physical diseases. The study findings suggest that African Americans' tendency to report more somatic symptoms is attributable to a reporting behavior or comorbidity rather than heightened somatic sensitivity. The study found no evidence for African Americans' underreporting of affective symptoms of depression, but this may be due to a small sample size and low statistical power.

The study sample size posts a major limitation to the interpretation of the study findings. When a study sample is as small as the White group in this study, a single extreme score can easily sway the group's mean score, making the difference between groups larger or smaller than expected. Therefore, findings from this study must be interpreted with caution and require replication with a larger study sample. A low statistic power associated with the small sample size limited the use of multivariate analysis. Study findings that were obtained from bivariate tests may be explained by demographic differences between groups rather than race. 
Table 2. Group Differences on Biological and Psychological Measures

\begin{tabular}{|c|c|c|c|c|c|}
\hline \multirow[t]{2}{*}{ Variable } & \multicolumn{2}{|c|}{ Depressed African Americans ( $n=15$ ) } & \multicolumn{2}{|c|}{ Depressed Whites $(\mathrm{n}=5)$} & \multirow[t]{2}{*}{$p$} \\
\hline & Mean & S.D. & Mean & S.D. & \\
\hline \multicolumn{6}{|l|}{ Biological measures } \\
\hline Serotonin $(\mathrm{ng} / \mathrm{mL})$ & 168.4 & 86.3 & 102 & 112.6 & .19 \\
\hline Morning cortisol & .23 & .13 & .42 & .19 & .036 \\
\hline Evening cortisol & .13 & .10 & .07 & .03 & .18 \\
\hline IL-6 (serum) & 2.7 & 1.8 & 2.2 & 1.6 & .55 \\
\hline \multicolumn{6}{|l|}{ Psychological measures } \\
\hline CES-D & 28.9 & 8 & 32.2 & 10.8 & .48 \\
\hline HAM-D & 15.1 & 5.1 & 20.4 & 13 & .19 \\
\hline BDI-II & 18.4 & 7.8 & 40.8 & 35.1 & .23 \\
\hline BSI & 55.9 & 18.2 & 62.8 & 37.1 & .60 \\
\hline BSI- depression & 6.9 & 4.1 & 7.8 & 4.6 & .67 \\
\hline BSI- somatization & 9.1 & 4.5 & 5.4 & 3.2 & .11 \\
\hline GBMMS & 30 & 6.6 & 27.4 & 3.3 & .42 \\
\hline GBMMS-suspicion & 14.1 & 4.7 & 8.8 & 2.8 & .032 \\
\hline
\end{tabular}

We also found that three White and two African American cancer patients used antidepressants to improve mood, while we had missing data on many others in this respect. The impact of antidepressants on psychological and biological measures of depression has yet to be understood. Despite these study limitations, this study provided intriguing results and some interesting trends, which warrant further investigation. It demonstrates the recruitment rate $(70 \%)$ and feasibility of conducting a biomarker study with minority cancer patients. It also provided preliminary data that may help with future sample size and power estimation.

\section{CONCLUSION}

The study findings are preliminary. They shed light on a discord between psychological and biological measures of depression, the usefulness of depression biomarkers and potential racial difference in the manifestation of depression. The implications are that nurses need to be cautious of somatic complaints that may be concomitants of other chronic conditions at the time of assessing African American cancer patients' depression. It is also important to keep in mind that cancer treatment brings about significant longterm side effects such as urinary incontinence, loss of sexual desire and erection dysfunction, which are physically taxing, psychologically stressful and yet too embarrassing to discuss. Nurses need to be able to talk openly with African American cancer patients about these issues to identify the source of somatic complaints and mental stress. Establishing trust is essential for accessing African American cancer patients' emotions and ensuring an accurate assessment of depression in this underserved patient population.

\section{ACKNOWLEDGEMENTS}

The study was supported by the National Institutes of Health/National Cancer Institute (R03 CA115191; PI:
Zhang; UL1 RR024989) and a pilot grant from the Comprehensive Cancer Center at Case Western Reserve University. Address correspondence regarding this article to Amy Y. Zhang, Ph.D., FPB School of Nursing, Case Western Reserve University, 10900 Euclid Avenue, Cleveland, $\mathrm{OH}$ 44106-4904.

\section{CONFLICT OF INTEREST}

None declared.

\section{REFERENCES}

[1] Satin JR, Linden W, Phillips M. Depression as a predictor of disease progression and mortality in cancer patients. Cancer 2009; 15: 5349-5361.

[2] Pinquart M, Duberstein PR. Depression and cancer mortality: A meta-analysis. Psychol Med 2010; 40: 1797-1810.

[3] ACS. Cancer Facts \& Figures for African Americans 2009-2010. Atlanta, GA: American Cancer Society, Inc. 2009.

[4] Brown C, Schulberg HC, Madonia MJ. Clinical presentations of major depression by African Americans and whites in primary medical care practice. J Affect Disord 1996; 41(3): 181-91.

[5] Giles DE, Perlis ML, Reynolds CF, Kupfer DJ. EEG sleep in African-American patients with major depression: a historical case control study. Depression Anxiety 1998; 8(2): 58-64.

[6] Kleinman A. Social origins of distress and disease : depression, neurasthenia, and pain in modern China. New Haven: Yale University Press 1986.

[7] Baker FM, Okwumabua J, Philipose V, Wong S. Screening African-American elderly for the presence of depressive symptoms: a preliminary investigation. J Geriatr Psychiatry Neurol 1996; 9(3): 127-32.

[8] Gary FA, Yarandi HN. Depression among southern rural African American women: A factor analysis of the Beck Depression Inventory-II. Nurs Res 2004; 53(4): 251-259.

[9] Brown C, Schulberg HC. Diagnosis and treatment of depression in primary medical care practice: the application of research findings to clinical practice. J Clin Psychol 1998; 54(3): 303-14.

[10] Brown C, Abe-Kim C, Barrio C. Depression in ethnically diverse women: Implications for treatment in primary care settings. Professional Psychol Res Pract 2003; 34(1): 303-314. 
[11] Raison CL, Miller AH. Depression in cancer: new developments regarding diagnosis and treatment. Biol Psychiatry 2003; 54(3): 283-94.

[12] Rief W, Pilger F, Ihle D, Bosmans E, Egyed B, Maes M. Immunological differences between patients with major depression and somatization syndrome. Psychiatry Res 2001; 105(3): 165-74.

[13] Newport DJ, Nemeroff CB. Assessment and treatment of depression in the cancer patient. J Psychosom Res 1998; 45(3): 215-37.

[14] Friedman MJ, Charney DS, Deutch AY. Neurobiological and clinical consequences of stress: from normal adaptation to posttraumatic stress disorder. Philadelphia: Lippincott-Raven Publishers 1995.
[15] Musselman, DL, Miller AH, Porter MR, et al. Higher than normal plasma interleukin-6 concentrations in cancer patients with depression: preliminary findings. Am J Psychiatry 2001; 158(8): $1252-7$.

[16] Luecken LJ, Compas BE. Stress, coping, and immune function in breast cancer. Ann Behav Med 2002; 24(4): 336-44

[17] Halbert CH, Weathers B, Delmoor E, et al. Racial differences in medical mistrust among men diagnosed with prostate cancer. Cancer 2009; 115 (11): 2553-61.

[18] Suite DH, La Bril R, Primm A, Harrison-Ross P. Beyond misdiagnosis, misunderstanding and mistrust: relevance of the historical perspective in the medical and mental health treatment of people of color. J Natl Med Assoc 2007; 99 (8): 879-85.

(C) Amy Y. Zhang; Licensee Bentham Open.

This is an open access article licensed under the terms of the Creative Commons Attribution Non-Commercial License (http://creativecommons.org/licenses/ by-nc/3.0/) which permits unrestricted, non-commercial use, distribution and reproduction in any medium, provided the work is properly cited. 